\title{
AN OPTIMIZATION MODEL TO MINIMIZE THE EXPECTED END-TO-END TRANSMISSION TIME IN WIRELESS MESH NETWORKS
}

\author{
Marlon da Silva $^{1 *}$, Edson L.F. Senne ${ }^{2}$ and Nandamudi L. Vijaykumar ${ }^{3}$ \\ Received August 01, 2016 / Accepted June 17, 2017
}

\begin{abstract}
Time metrics are extremely important to evaluate the transmission performance on Wireless Mesh Networks (WMNs), whose main characteristic is to use multihop technology to extend the network coverage area. One of such metrics is WCETT (Weighted Cumulative Expected Transmission Time), in which transmission times per hop are weighted for both proactive and reactive conditions. Furthermore, such metrics are able to detect delays that can degrade some network services. This paper presents an optimization model to minimize WCETT in a WMN, subject to constraints grouped by bandwidth, flow control and power control. As the model includes nonlinear constraints, we propose a heuristic to solve it, which divides the problem in two subproblems. The first subproblem maximizes the network link capacity and a Simulated Annealing algorithm is used to solve it. Considering the link capacities obtained, the second subproblem minimizes the WCETTs, which is formulated as a linear programming model. Some numerical results are presented, based on instances of WMNs randomly generated. Some of these results are compared with the results obtained by a commercial simulator in order to verify the coherence of the proposed heuristic for realistic scenarios.
\end{abstract}

Keywords: Wireless Mesh Networks, Mathematical Programming, WCETT, Cross-layer Optimization.

\section{INTRODUCTION}

Wireless networks are bringing fundamental changes in the access of network services, mainly due to the expressive use of portable devices, such as laptops, tablets, mobile phones etc. At the same time, multimedia (audio and video) services are commonly required by several network applications. These multimedia services, when accessed mainly by means of wireless communication, demand a good configuration of the network, such as a large coverage area and a very short transmission time.

\footnotetext{
*Corresponding author.

${ }^{1}$ CEMADEN - Centro Nacional de Monitoramento e Alertas de Desastres Naturais, Estr. Dr. Altino Bondesan, 500, Eugênio de Melo, 12247-016 São José dos Campos, SP, Brasil. E-mail: marlondasilv@ @otmail.com

${ }^{2}$ UNESP - Universidade Estadual Paulista, Av. Ariberto Pereira da Cunha, 333, Portal das Colinas, 12516-410 Guaratinguetá, SP, Brasil. E-mail: elfsenne@unesp.br

${ }^{3}$ INPE - Instituto Nacional de Pesquisas Espaciais, Av. dos Astronautas, 1758, Jd. da Granja, 12227-010 São José dos Campos, SP, Brasil. E-mail: vijay.nl@inpe.br
} 
Wireless Mesh Network (WMN) is an effective and cheap technology [1,11]. WMNs are being installed in residences, buildings, companies and vehicles due to their strength, self configuration and easy maintenance. WMNs are especially important where the installation of cables and fixed infrastructure is difficult.

The main characteristic of WMNs is the multihop technology, capable to relay data packets from some source to a particular destination in a hop-by-hop manner over the network devices. In a WMN, these network devices, known as Access Points (APs), are classified into two groups: gateways and routers. Gateways are the network devices connected directly to an external source (e.g., a wired network or a end-user equipment) and routers are APs which communicate with other APs in order to ensure the network's connectivity. So, in a WMN, the data routing involves not only the source and destination APs, but rather some relay APs as well, allowing data to be forwarded even if the path between source and destination is not permanently connected during communication.

Figure 1 shows an example of WMN and its respective links referring to the wireless connections between APs.

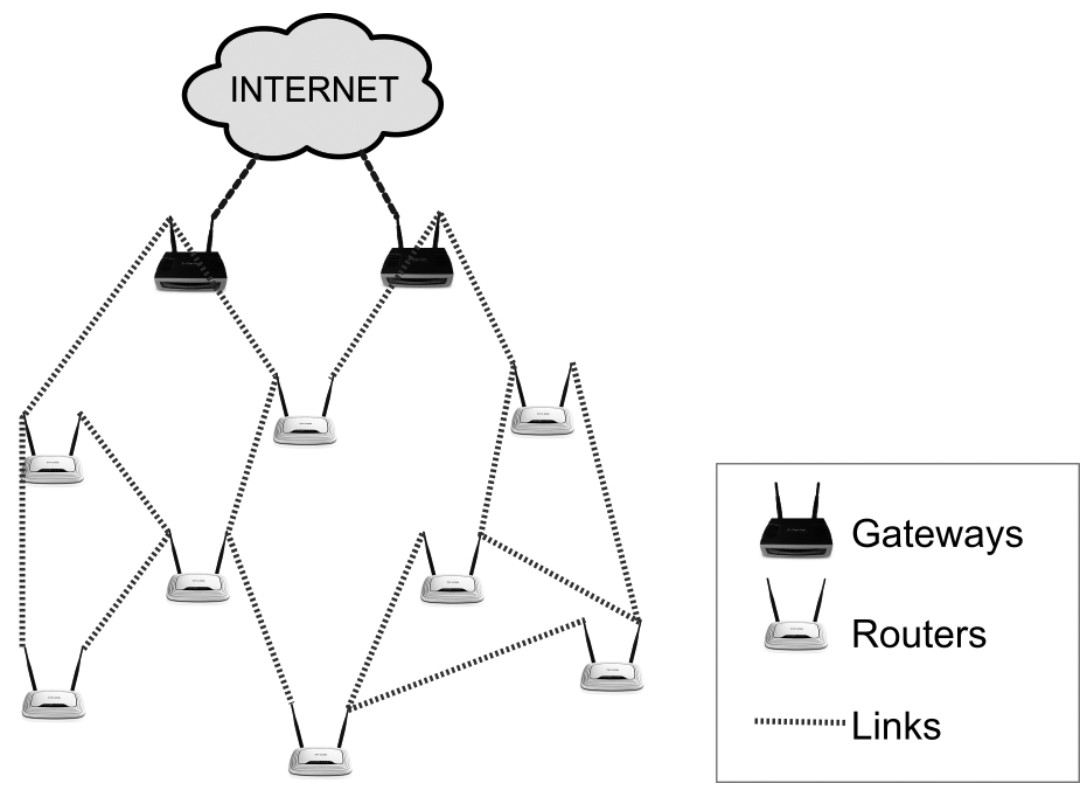

Figure 1 - An example of WMN, where black devices are gateways and white devices are routers.

The concept of sessions is an important issue in WMNs. A session is a set of links that compose a transmission path, from a gateway to a destination router. Figure 2 shows two sessions: the dashed links compose the path from gateway to the destination router A, and the dotted links compose the path from the gateway to the destination router B.

Wireless communication may suffer signal degradation due to some obstacles, such as buildings, noise from natural phenomena, or interference from other devices. There are some physical 


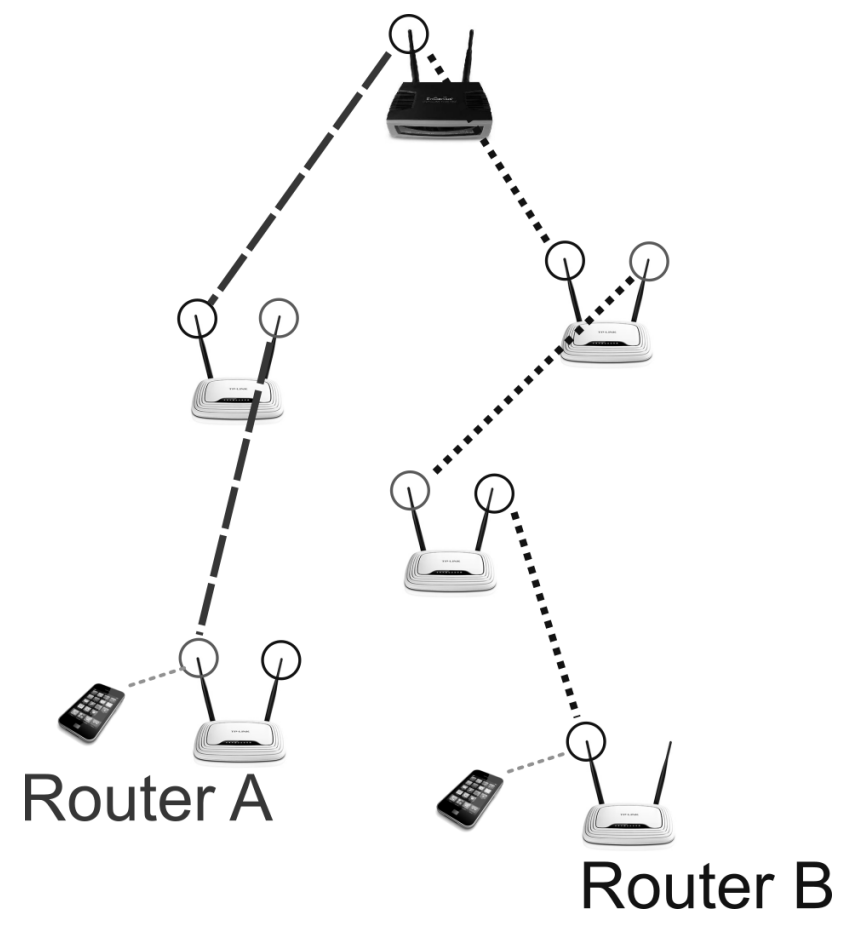

Figure 2 - Example of two sessions in a WMN.

models, such as SINR (Signal-to-Interference-plus-Noise Ratio) that enable estimating the relation between signal, noise and interference. This estimate provided by SINR model becomes important to determine the transmission capacity of each link in function of the consumed power not only by the link itself but also by its neighbor links $[9,20]$.

Due to its flexibility, a WMN requires a more careful planning in order to avoid inefficiencies that compromise the network performance and, consequently, discourage clients to use the network services. In general, the planning of a WMN involves building optimization models that take into consideration several aspects, such as flow control, power control, transmission rates, APs connectivity, signal interferences, etc. These aspects are related to different layers of the network architecture [22].

Metrics are also used to analyze and verify how good the service offered by the networks is. One of these most used metrics is the throughput [19,21]. Although very much used, throughput is not an appropriate metric to evaluate data routing. Therefore, one should consider metrics that consider evaluation of the transmission. For example, ETX (Expected Transmission Count) and ETT (Expected Transmission Time), consider the data path to evaluate the transmission during a session, enabling the determination of possible failures, such as loss of connection and bottlenecks. However, ETX and ETT are applied for each node individually and not to the entire path between the source node and the destination node [13]. So, one must consider other metrics that are able to accumulate the transmission times taken to traverse the entire path. 
WCETT (Weighted Cumulative Expected Transmission Time) is a metric that has been proposed as an improvement on ETT, which is especially important to evaluate WMNs performance [6]. WCETT is a routing metric that takes into account both link throughput and channel diversity. WCETT attempts to route packets using a path with the least number of nodes transmitting on the same channel in order to reduce intra-flow interference [10]. The calculation of WCETT involves various network components, such as link capacity, power level of each link and flow control. It is still an open problem of how to calculate WCETT in an efficient way with respect to costs [12].

This paper proposes an optimization model to minimize the sum of all WCETTs of a WMN, subject to constraints grouped by bandwidth, flow control and power control. This model enables obtain important values for planning a WMN, such as power level and capacity of each link and data flow, but includes nonlinear constraints. A heuristic is proposed to solve it, which divides the problem into two subproblems:

1. The first subproblem maximizes the network link capacity subject to power control and bandwidth constraints, and a Simulated Annealing algorithm is used to solve it;

2. Considering the link capacities obtained, the second subproblem minimizes WCETTs formulated as a linear programming model.

This paper is an improvement of the initial studies proposed in [16], in which link capacities were not considered. The main contribution of this paper is to include in the optimization model: (a) an estimate of the relation between signal and interference plus noise, and (b) routing metrics based on transmission time.

The paper is organized as follows. The main related papers available in the literature on the themes addressed in this work are reviewed in Section 2. Section 3 presents the mathematical model proposed to minimize WCETT. The proposed algorithm to solve the problem is presented in Section 4. Numerical results are presented in Section 5 and Section 6 presents some conclusions and possible future directions.

\section{RELATED PAPERS}

As commented before, the planning of a WMN involves several problems which occur at different levels of abstraction (known as layers) of the network architecture. Flow control, power control, channel assignment, signal interferences and routers connectivity, for example, are directly related to the tasks performed by the lower layers of the network architecture: physical layer, data-link layer, network layer and transport layer. By examining the communication tasks in these different layers one can obtain some measures of network performance. The amount of data processed in a given time interval (throughput) and the average delay in the data flow of a link, for example, are some of these measures. When some of these problems are handled together in a single model, then we have a cross-layer optimization model. 
An example of a cross-layer model can be seen in [19], in which the authors simultaneously considered channel allocation, routing, scheduling and power control problems, in order to maximize the network throughput. In this paper, the solutions were obtained by a heuristic applied firstly to the channel assignment problem and then to the others.

The presence of noise and interference is an important factor in estimating the power levels that each access point must emit and thus, the use of physical models to deal with noise and interference becomes essential for the network planning. Some efforts using the physical model SINR have been studied in [17] to verify the link capacities in a WMN under different environmental conditions, in order to improve the quality of service offered by the network.

In [22], a WMN is modeled as a graph, in which each link has two different weights: data flow and power level. The authors propose a model for throughput maximization and show that the problem can be decomposed into two subproblems: a data routing subproblem (at the network layer) and a power control subproblem (at the physical layer). The physical model SINR is considered. Several effective solutions are discussed for each subproblem.

A cross-layer optimization for WMN is also considered in [21] taking into account APs with multiple antennas in order to maximize throughput and minimize the system activation time. Smart antenna techniques are used to provide interference suppression, capability for simultaneous communication with several nodes and transmission with higher data rates. Three MILP models are presented and the optimal solution is approached by a decomposition method.

In [15] is considered the impact of advanced physical layer techniques on the maximum achievable throughput of WMNs. A cross-layer optimization model goal is formulated for the routing and scheduling problem jointly to verify the impact of signal degradation on throughput. The model is solved by a Column Generation method and the solution found is used to establish the min-max levels to guarantee an acceptable throughput. However, link capacities are not considered and the proposed model considers that all APs have the same power level.

Cross-layer model is also considered in [2] for MIMO-based WMNs. Multiple-In-Multiple-Out) (MIMO) technology can bring substantial throughput improvement in WMNs by combining interference suppression and spatial multiplexing. The model involves end-to-end rate allocation, routing, and stream control scheduling in order to find the maximum throughput, max-min fairness and proportional fair rate allocation solutions. By using a greedy heuristic algorithm, the model has shown effective for WiMAX networks.

All the above mentioned works use heuristic methods to find good solutions for the problems due to the high complexity of the optimization models. In general, cross-layer optimization models are difficult to solve since they involve different problems. Such problems, even when isolated, are complex to solve. In particular, for WMNs, when it is necessary to consider each hop from the source gateway to the destination routers, such as proposed in this paper for minimizing WCETT, the complexity increases. 


\section{THE PROPOSED MODEL}

To estimate the transmission time in WMNs, routing metrics, such as ETX and ETT, are determined. These metrics must be measured during network running time [7]. ETX [10] represents the number of times that an AP waits to send or resend a data packet to a receiver device without compromising the packet. ETT is the average time a data packet travels on a link [4], which depends on the packet size $(\lambda)$ and link capacity $(\kappa)$. Hence, ETT can be calculated as: $E T T=E T X \times \frac{\lambda}{\kappa}$.

The total sum of ETTs is an estimate of the end-to-end delay experienced by a packet traveling along a path. However, ETT only does not consider any bottleneck on channel traffic. On the other hand, WCETT measures the cumulative time of the wireless hops to convey information from a source to a destination, considering possible bottlenecks on channels during the transmission [6]. So, WCETT can be considered as a good quality of service (QoS) parameter for WMNs, since the calculation of WCETT involves the evaluation of the entire path of all data packets [5].

In order to calculate WCETT along a path, it is not enough simply adding up ETTs along that path because this will not consider the impact of channel diversity, that is, will not distinguish between hops that are on different channels. One must remember that if two hops on a path are on the same channel then they always interfere with one another. Consider an $n$-hop path. Assume that the network has a total of $C$ channels. Let $\mathbf{H}$ be the set of hops that compose this path. Define $X_{j}$ as:

$$
X_{j}=\sum_{i \in \mathbf{H} \text { using } j}^{n} E T T_{i} \quad 1 \leq j \leq C
$$

Thus, $X_{j}$ is the sum of transmission times of hops on channel $j$. The total path throughput will be dominated by the bottleneck channel, which has the largest $X_{j}$.

So, in order to establish a tradeoff between delay and throughput, WCETT on a path can be calculated by:

$$
W C E T T=(1-\beta) \sum_{i \in \mathbf{H}} E T T_{i}+\beta \max _{1 \leq j \leq C} X_{j}
$$

where $\beta(0 \leq \beta \leq 1)$ is a tuning parameter, which corresponds to the percentage of the path that has to be dynamically built. Note that in (2), the first term can be considered as a measure of the latency of this path and the second term can be viewed as a measure of path throughput, since it represents the impact of bottleneck hops.

The model proposed in this paper has as objective to find a network configuration which minimizes the sum of the WCETTs in a WMN, subject to constraints grouped by the following subproblems: flow control, power control and link transmission capacities (bandwidth). These subproblems are directly related to the tasks within the physical, data-link, network and transport layers [3] and, therefore, this proposed model can be considered as a cross-layer optimization model. 
The main difference between the proposed model in this paper with the other models found in the literature is that the proposed model uses WCETT as a routing metric. This specific metric enables the evaluation of end-to-end paths considering possible bottlenecks on the channels, besides dealing with some effects of noise and interference in wireless transmission.

Table 1 shows the list of sets and subsets that will be used in the proposed model.

Table 1 - List of sets used in this model.

\begin{tabular}{c|l}
\hline Notation & Description \\
\hline $\mathbf{N}$ & Set of nodes (APs) \\
$\mathbf{N}^{s}$ & Set of nodes which are sender APs \\
$\mathbf{N}^{r}$ & Set of nodes which are receiver APs \\
\hline $\mathbf{S}$ & Set of sessions, where $\mathbf{S}=\{1, \ldots, S\}$ \\
\hline $\mathbf{L}$ & Set of links \\
$\mathbf{L}_{k}$ & Subset of links for session $k \in \mathbf{S}$ \\
$\mathbf{L}_{n}^{s}$ & Subset of links, where $n \in \mathbf{N}$ is a sender AP \\
$\mathbf{L}_{k n}^{s}$ & Subset of links, where $n \in \mathbf{N}$ is the sender AP of session $k \in \mathbf{S}$ \\
$\mathbf{L}_{k n}^{r}$ & Subset of links, where $n \in \mathbf{N}$ is the receiver AP of session $k \in \mathbf{S}$ \\
\hline
\end{tabular}

Let $(\mathbf{N}, \mathbf{L})$ be a graph which denotes a WMN, given $\mathbf{N}$, the set of APs, and $\mathbf{L} \subset \mathbf{N} \times \mathbf{N}$, the set of links. A session $k \in \mathbf{S}$ is denoted by a triple $\left(n_{k}^{s}, n_{k}^{r}, \lambda_{k}\right)$, where $n_{k}^{s}$ is the sender AP (usually, a gateway), $n_{k}^{r}$ is a receiver AP and $\lambda_{k}$ is the packet size of the session $k$. Figure 3 shows a graph representation of a WMN, where the vertices represent APs (gateways, relay routers or destination routers) and the edges represent the links.

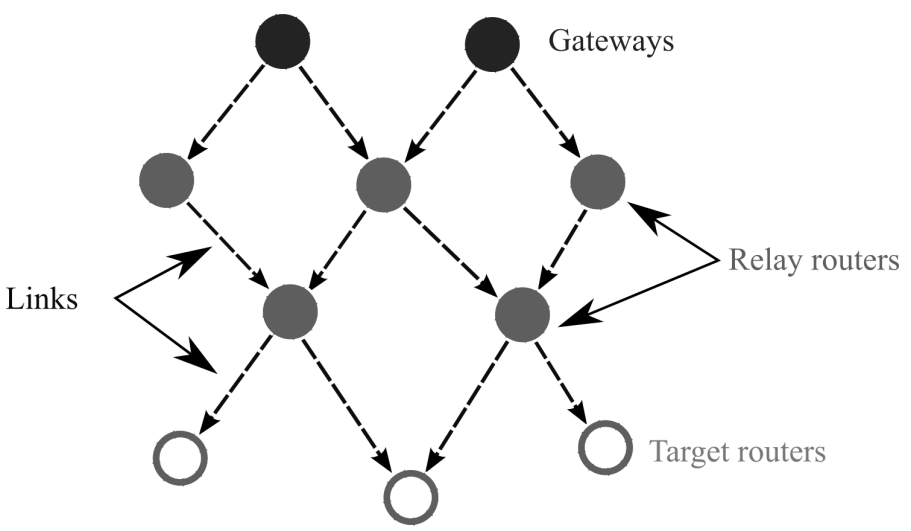

Figure 3 - A graph representation of a WMN.

Table 2 shows the variables and Table 3 lists the parameters used in the proposed model.

In the proposed model, all ETXs are considered as probabilistic parameters [6]. Coefficient $\beta$ refers to an important characteristic of the network: if $\beta<0.5$, the network will prioritize 
Table 2 - Variables used in the model.

\begin{tabular}{|c|c|c|}
\hline Variable & Type & Description \\
\hline$\kappa_{j}$ & Non-negative real & Link capacity \\
\hline$p_{j}$ & Non-negative real & Power consumed by link $j$ \\
\hline$f_{j}^{k}$ & Non-negative real & $\begin{array}{l}\text { Data flow that travels on each link } j \in \mathbf{L} \text { in the session } \\
k \in \mathbf{S}\end{array}$ \\
\hline$\tau_{j}^{k}$ & Non-negative real & ETT of each link $j \in \mathbf{L}$ in the session $k \in \mathbf{S}$ \\
\hline$t$ & Non-negative real & Maximum transmission time per hop \\
\hline
\end{tabular}

Table 3 - Parameters used in the model.

\begin{tabular}{c|l}
\hline Parameter & Description \\
\hline$\beta$ & Tunable parameter to evaluate WCETT [6] \\
$G_{m n}$ & Array element of interference coefficient between APs $m$ and $n$ \\
$\sigma$ & Noise coefficient \\
$P_{n}$ & Maximum power of a AP $n \in \mathbf{N}$ \\
$\chi_{j}^{k}$ & ETX, given by a probabilistic parameter \\
$W$ & Bandwidth of each frequency band \\
\hline
\end{tabular}

proactive routing features, emphasizing paths previously determined. Otherwise, the network will prioritize reactive features, emphasizing the path construction on demand $[5,6]$. The values for interference coefficients $G_{m n}$ are calculated in function of the distance between APs $m$ and $n$ $\left(d_{m n}\right)$ and path loss exponent $(\eta)[19]$, i.e.:

$$
G_{m n}=d_{m n}^{-\eta}
$$

The proposed model is presented as follows:

$$
\min \sum_{k=1}^{S}\left[(1-\beta) \sum_{j \in \mathbf{L}_{k}} \tau_{j}^{k}+\beta t\right]
$$

Subject to:

$$
\begin{array}{ll}
t \geq \sum_{j \in \mathbf{L}} \sum_{k=1}^{S} \tau_{j}^{k} & \\
\sum_{p \in \mathbf{L}_{k n}^{s}} f_{p}^{k}-\sum_{q \in \mathbf{L}_{k n}^{r}} f_{q}^{k}=0 & \forall k \in \mathbf{S} ; n \in \mathbf{N}-\left\{n_{k}^{s}, n_{k}^{r}\right\} \\
\sum_{p \in \mathbf{L}_{k n}^{s}} f_{p}^{k}-\sum_{q \in \mathbf{L}_{k n}^{r}} f_{q}^{k}=\lambda_{k} & \forall k \in \mathbf{S} ; n \in \mathbf{N}^{s}
\end{array}
$$




$$
\begin{array}{ll}
\sum_{p \in \mathbf{L}_{k N}^{s}} f_{p}^{k}-\sum_{q \in \mathbf{L}_{k N}^{r}} f_{q}^{k}=-\lambda_{k} & \forall k \in \mathbf{S} ; n \in \mathbf{N}^{r} \\
\sum_{j \in \mathbf{L}_{n}^{s}} p_{j} \leq P_{n} & \forall n \in \mathbf{N} \\
\sum_{k=1}^{S} f_{j}^{k} \leq \kappa_{j} & \forall j \in \mathbf{L} \\
\kappa_{j}=W \log _{2}\left(1+\frac{G_{m n} p_{j}}{\sigma+\sum_{(u, v)=\theta \in \mathbf{L} ; \theta \neq j} G_{u n} p_{\theta}}\right) & \forall[j=(m, n)] \in \mathbf{L} \\
\tau_{j}^{k}=\frac{\chi_{j}^{k} \lambda_{k}}{\kappa_{j}} & \\
\tau_{j}^{k} \geq 0, f_{j}^{k} \geq 0 & j \in \mathbf{L} ; k \in \mathbf{S} \\
p_{j} \geq 0, \kappa_{j} \geq 0 & \forall j \in \mathbf{L} ; k \in \mathbf{S}
\end{array}
$$

The objective function (4) refers to minimizing the sum of all WCETTs in the WMN.

Constraint (5) represents the reactive features of the routing protocol. This constraint ensures the largest value for the sum of ETTs, in order to avoid bottleneck in the paths [6].

Constraints (6-8) guarantee the flow balance in the WMN: constraint (6) ensures that the received data in an relay router is equal to respective transmitted data. Constraints (7) and (8) indicate, respectively, the data flow for sender and receiver APs.

Constraint (9) ensures a power limit that an AP $n \in \mathbf{N}$ can emit to their neighbor devices, i.e., the sum of power level of all links where $n$ is the sender cannot be greater than the maximum AP power $P_{n}$.

Bandwidth constraints improve data flow in WMNs, considering the transmission capacity of each link [21]. Constraint (10) ensures that the total data flow on a link cannot be greater than the link capacity. Constraint (11) determines the link capacity using Shannon equation in function of a transmission model that considers the signal from the sender AP, the interference of other APs and the environment noise. In this case, the model adopted is Signal-to-Interference-plus-Noise Ratio (SINR).

Constraint (12) ensures the value for each ETT in function of the respective ETX $\chi_{j}$, the data size packet $\lambda_{k}$ and the link capacity $\kappa_{j}$.

Constraints (13) and (14) limit the values assigned to the decision variables.

The next section presents a heuristic to find good solutions for this model. The idea of this heuristic is to solve the non-linear part of the model using the metaheuristic Simulated Annealing $[14,18]$, and then, obtain a solution for the problem by employing a linear programming model. 


\section{HEURISTIC TO OBTAIN WCETT}

To obtain a solution for the proposed model in an acceptable computational time, we propose a hybrid heuristic which decomposes the problem into two subproblems:

1. Maximization of link capacities. This subproblem consists of finding the power levels and link capacities, in order to maximize the total capacity of the links;

2. Minimization of WCETTs. This subproblem corresponds to the Linear Programming model which arises when constraints (9) and (11) are removed from the proposed model, given the values for link capacities obtained in the previous subproblem.

\subsection{Maximization of link capacities}

The first step of proposed heuristic consists in finding power levels for each link, in order to maximize the total link capacity. A similar strategy is also applied in [22]. One may observe that the link capacity must be obtained in function of the power of the link itself and the power of neighbor links that can cause interference. This estimation is used to avoid any large discrepancy in power link values and, consequently, in link capacities. Hence, the maximization of link capacities is formulated considering power control and link capacity constraints as a function of SINR. Thus, this subproblem is formulated as follows:

$$
\max \sum_{j=1}^{L} \kappa_{j}
$$

Subject to:

Constraints (9), (11), (14).

In this subproblem, the main idea is to maximize the link capacity of WMN according to SINR measured in the physical layer of the network. To solve this subproblem, we employ the Simulated Annealing metaheuristic shown in Algorithm 1.

Some parameters are used in Simulated Annealing algorithm. In particular, let $\mathbf{p}_{0}$ be the initial set of power levels used to estimate all link capacities of the WMN. Furthermore, let $T_{0}$ be the initial parameter that controls the perturbation of the current solution (known as temperature) and $\epsilon$ the minimum temperature. For each temperature $T$, a solution is chosen and this solution may be better than the current best solution, denoted by $\mathbf{p}_{\text {best }}$. If the best solution is not improved, the chosen solution may still be accepted, according to values of $T$ as well as the difference between values of the objective function assigned to neighbor solutions, denoted by $\Delta f$. The value of $T$ is updated and this procedure is repeated until $T$ becomes less than $\epsilon$ due to cooling rate $\alpha$ whose range is $0<\alpha<1$.

Note that, to choose a neighbor solution, the Simulated Annealing algorithm invokes the function Find_Neighbor(p), described in Algorithm 2.

Once the best values for each link capacity $\kappa_{j}(j \in \mathbf{L})$ are obtained, they will be used as parameters to the next subproblem, so that the resulting model becomes linear. 


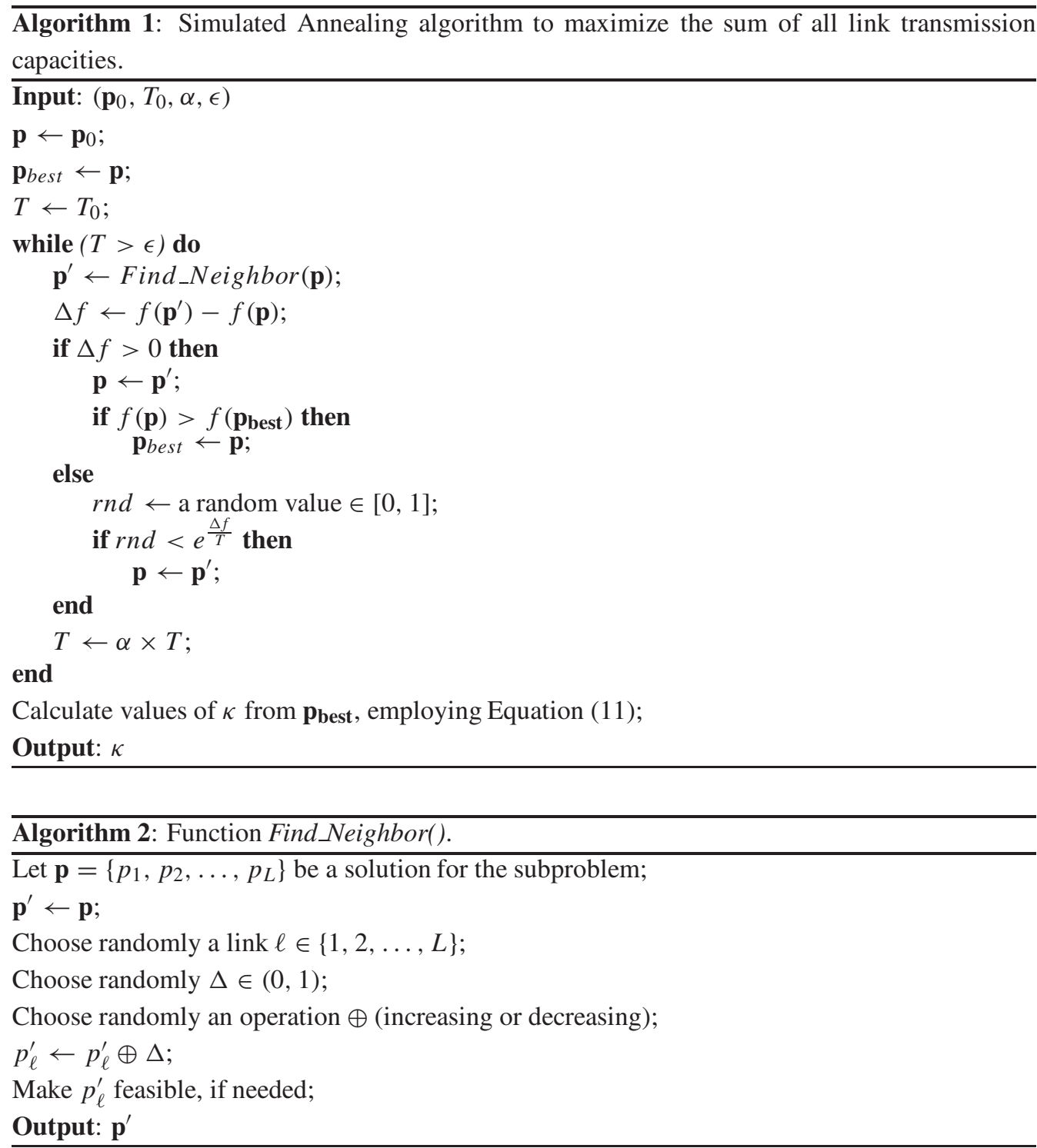

\subsection{Minimization of WCETTs}

Once link capacities and link power levels have been obtained, the second step of the proposed heuristic is to determine the values of flow control and ETT. Thus, the subproblem to minimize WCETTs is formulated as follows:

$$
\min \sum_{k=1}^{S}\left[(1-\beta) \sum_{j \in \mathbf{L}^{k}} \tau_{j}^{k}+\beta t\right]
$$


Subject to:

Constraints (5-8), (10), (12) and (13).

This subproblem can be solved by a commercial solver. Its solution represents the data flow and the ETTs of each link that minimize the total transmission time of the WMN.

\section{NUMERICAL RESULTS}

The proposed heuristic was tested on instances representing WMNs in a grid topology over an outdoor environment where neighbor APs are distant 50 meters from each other. The algorithm was written in C++ using libraries of the IBM CPLEX 12.5 solver [8] and run on a microcomputer with quad-core $2.5 \mathrm{GHz} \mathrm{CPU}$ and 4 GB RAM.

\subsection{Tests using a default instance}

In this section, we consider a $3 \times 3$ grid topology and the following default parameters:

1. (Number of APs) $N=9$, where 1 is the gateway, 7 are relay routers and 1 is the destination AP;

2. (Number of sessions) $S=6$;

3. (ETX) $\chi_{j}^{k}=1.05$;

4. (Tuning coefficient) $\beta=0.7$;

5. (Demand rate) $\lambda^{k}=4 \mathrm{Mb} / \mathrm{s}$ per session;

6. (Maximum power used by router) $P_{n}=100 \mathrm{~mW}$;

7. (Noise coefficient) $\sigma=-80 \mathrm{dBm}=10^{-8} \mathrm{~W}$;

8. (Bandwidth frequency) $W=20 \mathrm{MHz}$ per channel;

9. (Path loss exponent) $\eta=3$.

For the Simulated Annealing algorithm, after a parameter tuning process where were considered: initial temperature in $[100,100000]$, cooling rate in $[0.5,0.95]$ and minimum acceptable temperature in $[0.1,0.001]$, the following values were chosen:

1. (Initial temperature) $T_{0}=10000$;

2. (Cooling rate) $\alpha=0.9$;

3. (Minimum acceptable temperature) $\epsilon=0.001$. 
Table 4 - Results obtained for default instance - Subproblem 1.

\begin{tabular}{l|c}
\hline Description & Value \\
\hline Average solution $(\mathrm{Mb} / \mathrm{s})$ & 832.00 \\
Best solution $(\mathrm{Mb} / \mathrm{s})$ & 866.29 \\
Average deviation $(\%)$ & 1.54 \\
Computational Time (s) & 21.65 \\
\hline
\end{tabular}

For the tests, the Simulated Annealing algorithm was executed 100 times. Table 4 shows the obtained values. In this table, the average deviation corresponds to the ratio between the average of standard deviations and the average solution.

Table 5 shows the results obtained by CPLEX solver, considering the link capacities obtained previously.

Table 5 - Results obtained for default instance - Subproblem 2.

\begin{tabular}{l|l}
\hline Description & Value \\
\hline Total WCETT (s) & 0.146 \\
Average WCETT per session (s) & 0.024 \\
Average ETT (ms) & 1.787 \\
\hline
\end{tabular}

Figure 4 illustrates the averages of each link capacity and ETT per hop in the grid. Note that the Simulated Annealing algorithm obtained average link capacities greater for links that are closer to the gateway in relation to the other links. This result matches with the physical model of the network, because interference is greater when there is a significant number of devices around the link signal. Hence, the lowest values for ETTs correspond to the links that contain the highest link capacities.

\section{Capacity per link $(\mathrm{Mb} / \mathrm{s})$}

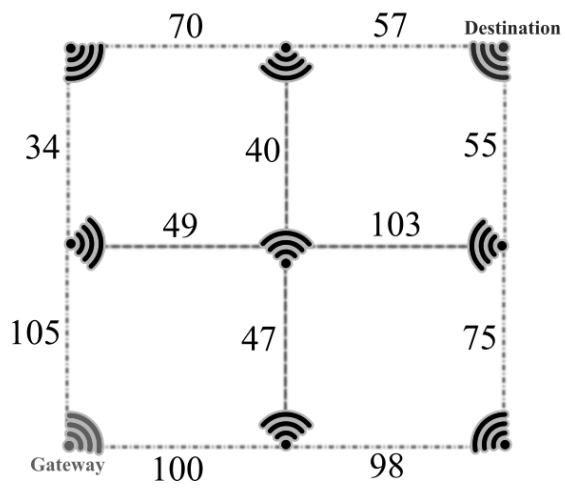

ETT per link (ms)

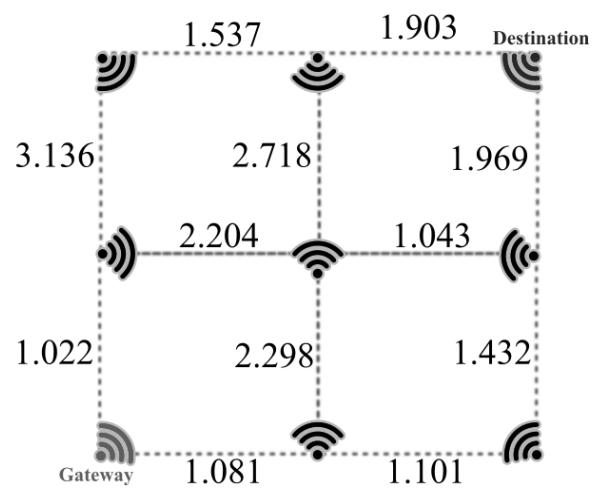

Figure 4 - Average link capacities and ETTs over a WMN grid $3 \times 3$. 


\subsection{Tests using other instances}

Tests with other instances were conducted to verify the behavior of the proposed heuristic when the grid size increases. These instances differ from the default instance in the grid size and, consequently, in the number of APs. The position of the gateway and the destination AP will be the same in all instances: the gateway is located at the bottom-left corner and the destination AP is at the top-right corner of the grid. Figure 5 illustrates $4 \times 4$ network sample and its respective gateway and destination AP positions.

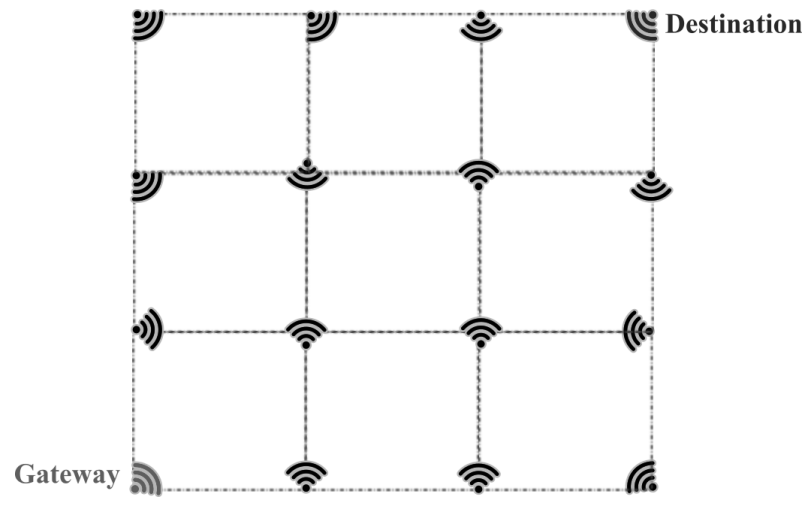

Figure 5 - Gateway and AP destination positions in the WMN grid $4 \times 4$.

The number of sessions is not important in this case and was defined, arbitrarily, as $S=4$ for all these instances. Table 6 shows the results obtained for these network samples in terms of total WCETT, average ETT and processing time.

Table 6 - Results obtained varying grid size.

\begin{tabular}{c|c|c|c|c}
\hline Grid size & Links per session & Total WCETT $(\mathrm{s})$ & Average ETT $(\mathrm{ms})$ & CPU time $(\mathrm{s})$ \\
\hline $4 \times 4$ & 6 & 0.249 & 2.24 & $<1$ \\
$6 \times 6$ & 10 & 0.518 & 2.82 & $<1$ \\
$8 \times 8$ & 14 & 0.999 & 3.20 & $<1$ \\
$12 \times 12$ & 22 & 2.493 & 3.72 & $<1$ \\
$25 \times 25$ & 48 & 32.095 & 6.43 & 2 \\
$36 \times 36$ & 70 & 96.152 & 9.28 & 10 \\
$50 \times 50$ & 98 & 239.433 & 11.97 & 36 \\
\hline
\end{tabular}

Figure 6 illustrates the evolution of total WCETT, while Figure 7 shows the evolution of average ETT.

As one can notice, Total WCETT, Average ETT, and the computational time increase when the number of APs increases, as expected. The growth of the total WCETT is justified due to the growth in the number of links per session but, mainly due to the exponential increase in the 


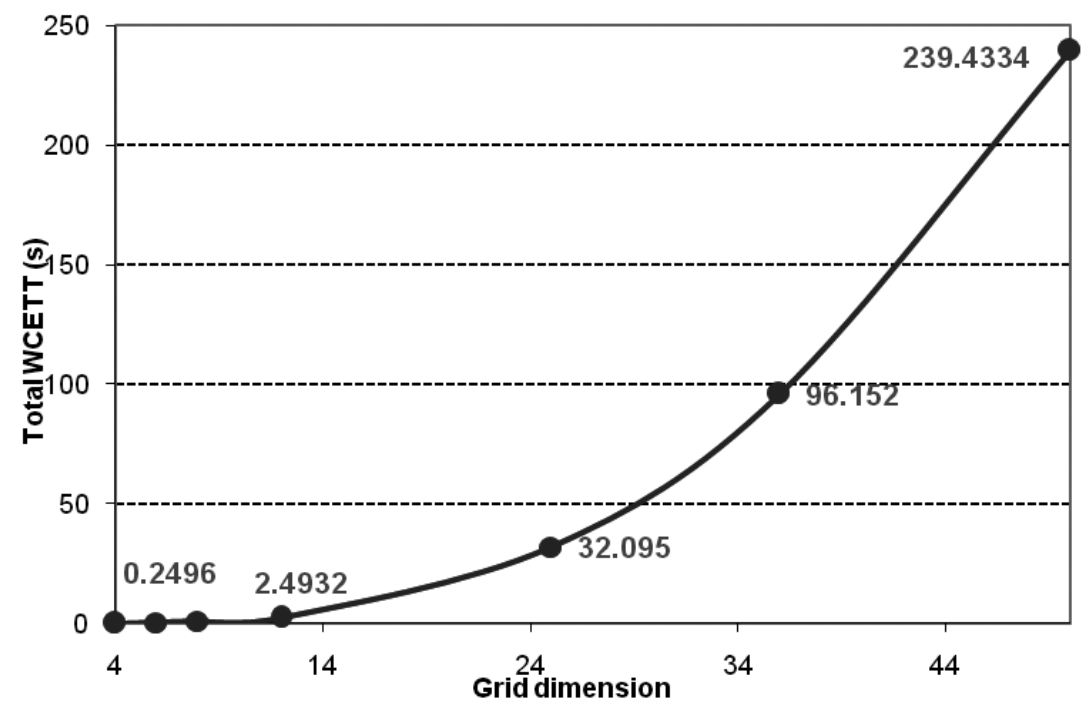

Figure 6 - Total WCETT in function of grid size.

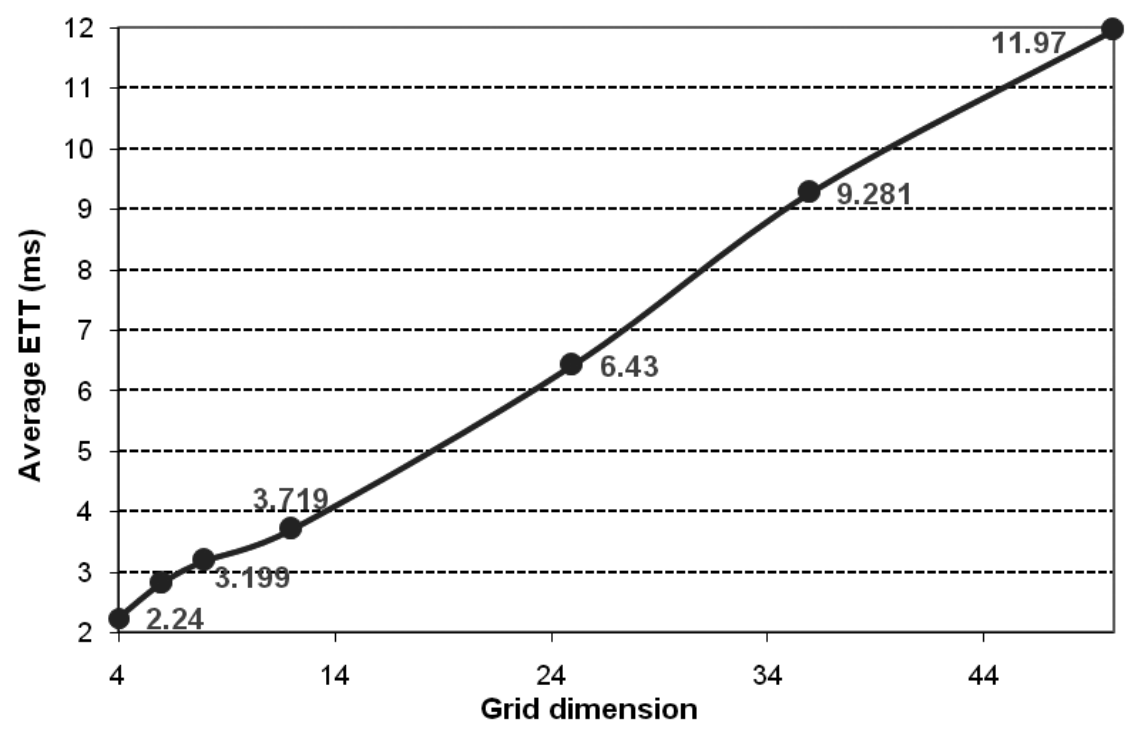

Figure 7 - Average ETT in function of grid size.

number of possible paths (possible hops) in the network, from the gateway to the destination. In addition, average ETT has increased due to an increase in the number of wireless links to be included in the path. As a consequence, there are more noise and interference from other devices during the traverse. With respect to the computational time, the proposed heuristic showed to be fast, even for larger networks, such as with a grid size of $50 \times 50$. 


\subsection{Tests modifying the number of sessions}

Some tests were conducted on the default instance, modifying only the number of sessions. The objective of these tests is to evaluate the network behavior when data traffic increases. Sessions were created randomly and the sender AP is always the gateway. Table 7 shows the results obtained for Total WCETT and Figure 8 presents its behavior in function of the number of sessions.

Table 7 - Results obtained from the number of sessions.

\begin{tabular}{c|c}
\hline Sessions & Total WCETT $(\mathrm{s})$ \\
\hline 4 & 0.1039 \\
8 & 0.2077 \\
12 & 0.3806 \\
16 & 0.4913 \\
24 & 0.6082 \\
\hline
\end{tabular}

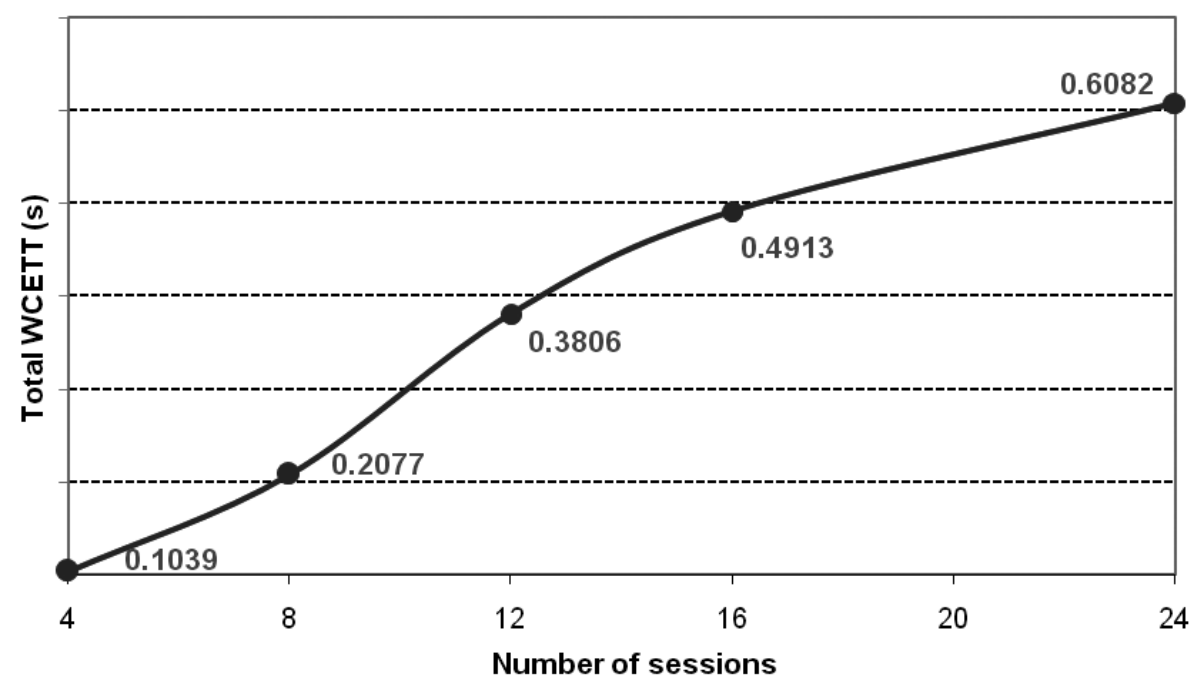

Figure 8 - Total WCETT in function of the number of sessions.

From these results, it is possible to notice that the number of sessions does not impact significantly on average ETT and the increase in the values of total WCETT is smooth.

\subsection{Comparison with Network Simulator 3}

The results obtained by the proposed heuristic were compared to values found by NS-3, a simulator oriented to wireless networks. These necessary tests were conducted with an objective to verify the coherence of the proposed heuristic for realistic scenarios. 
It is important to highlight that the heuristic proposed in this paper determines ETT on each link and, then, computes the WCETT. NS-3 simulator is not able to calculate ETTs on an individual basis and, moreover, NS-3 does not have the protocol MR-LQSR, a key protocol used to determine WCETT. Therefore, the results returned by NS-3 are obtained from non-optimized protocols.

The simulation was executed 50 times. The total data transmission time is $900 \mathrm{~s}$ and the interval between two consecutive packets is $10 \mathrm{~ms}$. Table 8 shows the values for average ETT and total WCETT obtained by the proposed heuristic as well as by the simulator.

Table 8 - Results obtained from heuristic and compared with simulation.

\begin{tabular}{c|c|c|c|c}
\hline & \multicolumn{2}{|c|}{ Total WCETT $(\mathrm{s})$} & \multicolumn{2}{c}{ Average ETT $(\mathrm{ms})$} \\
\hline Grid size & Heuristic & NS-3 & Heuristic & NS-3 \\
\hline $3 \times 3$ & 0.102 & 0.128 & 1.894 & 1.354 \\
$4 \times 4$ & 0.249 & 0.305 & 2.261 & 1.598 \\
$5 \times 5$ & 0.351 & 0.655 & 2.546 & 4.049 \\
\hline
\end{tabular}

Table 8 shows that the proposed heuristic obtained lower values for total WCETT when compared to the values obtained by NS-3. These results show that the heuristic is capable to recommend a better network configuration in terms of transmission times. However, the heuristic does not consider details of configuration which are considered by the simulator, such as type of technology used in each layer, protocols, etc. Furthermore, the simulation dynamically calculates the packet loss, while the heuristic considers ETX as a parameter to obtain other metrics.

It is important to observe that the simulator NS-3 was not able to obtain results for grid sizes greater than $5 \times 5$.

\section{CONCLUSIONS}

This paper presented an optimization model applied to WMNs in order to minimize the time routing metric WCETT, considering some important problems found in networks, such as bandwidth usage, flow control and power control. In WMNs these problems are essential to be sorted out in order to ensure the connectivity between APs which is necessary for the multihop technology.

The proposed model contributes to obtain a good configuration for WMNs, which can be crucial if applications that do not accept large delays (such as multimedia applications) are extensively used in the network. The work also proposes a heuristic to solve the model. Computational tests show that the proposed heuristic is efficient and obtains satisfactory results, when checked by a network simulator.

This research can contribute for the diffusion of the WMN technology, which can be important in places where infrastructure is scarce, such as in remote locations in the Amazon region. Wellplanned WMNs can offer good quality network services without affecting the environment. 
There are some possibilities to be explored as future work, such as the inclusion of other problems in the optimization model, for example, channel assignment. Furthermore, other metaheuristics such as Variable Neighborhood Search and Iterated Local Search could be used to solve the link capacity problem. An interesting research issue could be to come up with a heuristic to solve the entire problem without the need to decompose it into subproblems.

\section{ACKNOWLEDGEMENTS}

The authors acknowledge Brazilian agencies National Council for Research and Scientific Development $(\mathrm{CNPq})$ and the Coordination for the Improvement of Higher Education Personnel (CAPES) within Academic Cooperation Project (PROCAD - Grant 776/2010). The authors are also grateful to the anonymous reviewers for their valuable comments and useful suggestions that have significantly improved this paper. The first author acknowledges Ph.D. Diego Herbín Stalder Díaz for his kind support in this work.

\section{REFERENCES}

[1] AKYILDIZ IF, WANG X \& WANG W. 2005. Wireless mesh networks: a survey. Computer Networks ISDN Systems, 47(4): 445-487, mar.

[2] Bansal M \& TRivedi A. 2014. Cross-layer optimization for fair end-to-end rate allocation in WMNs with MIMO links. Transactions on Emerging Telecommunications Technologies, 25(5): 496506.

[3] Blank AG. 2004. TCP/IP Foundations. Sybex.

[4] Borges VCM, Curado M \& Monteiro E. 2010. A cross-layer routing scheme for scalable triple play service in wireless mesh networks. In Computer Communications and Networks (ICCCN), 2010 Proceedings of 19th International Conference on, pages 1-6, aug.

[5] Campista M, Esposito P, Moraes i, Costa L, Duarte O, Passos D, Albuquerque C, SAADE D \& RUBINSTEIN M. 2008. Routing metrics and protocols for wireless mesh networks. Network, IEEE, 22(1): 6-12, jan.-feb.

[6] Draves R, PAdhye J \& ZiLl B. 2004. Routing in multi-radio, multi-hop wireless mesh networks. In Proceedings of the 10th annual international conference on Mobile computing and networking, MobiCom '04, pages 114-128, New York, NY, USA, ACM.

[7] Esposito P, Campista M, Moraes I, Costa L, Duarte O \& Rubinstein M. 2008. Implementing the expected transmission time metric for OLSR wireless mesh networks. In Wireless Days, 2008. WD '08. 1st IFIP, pages 1-5, nov.

[8] IBM ILOG CPLEX. 2010. V12.5: User's Manual for CPLEX. International Business Machine Corporation,

[9] Khreishah A, WANG C-C \& ShrofF N. 2009. Cross-layer optimization for wireless multihop networks with pairwise intersession network coding. Selected Areas in Communications, IEEE Journal on, 27(5): 606-621.

[10] LAVÉN A \& HJÄRTQUiST P. 2009. Multimetric OLSR and ETT. In 5th OLSR Interop \& Workshop, Vienna. 
[11] Lee M, Zheng J, Ko Y-B \& Shrestha D. 2006. Emerging standards for wireless mesh technology. Wireless Communications, IEEE, 13(2): 56-63, april.

[12] Misra S, Misra SC \& Woungang I. 2009. Guide to wireless mesh networks. Springer.

[13] Parissidis G, Karaliopoulos M, Baumann R, Spyropoulos T \& Plattner B. 2009. Routing metrics for wireless mesh networks. In MisRa S, MisRa S \& Woungang I (editors). Guide to Wireless Mesh Networks, Computer Communications and Networks, pages 199-230. Springer London.

[14] Roeva O \& Trenkova T. 2012. Modelling of a fed-batch culture applying simulated annealing. BIOMATH, 1(2), dec. 2012.

[15] Shabdanov S, Mitran P \& Rosenberg C. 2012. Cross-layer optimization using advanced physical layer techniques in wireless mesh networks. Wireless Communications, IEEE Transactions on, 11(4): 1622-1631, april.

[16] Silva M, Senne ElF \& Vijay kumar NL. 2014. Cross-layer optimization applied to obtain time metric specified to wireless mesh networks. In Proceedings of 11th International Conference on Operations Research, pages 1-4, Havana.

[17] Silva M, SENNE ELF \& ViJAY KUMAR NL. 2014. Optimization methods applied to nonlinear signal interference models. In Proceedings of 4th International Conference on Engineering Optimization, volume 1, pages 681-686, Lisbon.

[18] SzU H \& HARTLEY R. 1987. Nonconvex optimization by fast simulated annealing. Proceedings of the IEEE, 75(11): 1538-1540.

[19] TANG J, Xue G \& Zhang W. 2009. Cross-layer optimization for end-to-end rate allocation in multi-radio wireless mesh networks. Wireless Networks, 15(1): 53-64, Jan.

[20] Weber S \& Andrews JG. 2012. Transmission capacity of wireless networks. The Computing Research Repository, abs/1201.0662.

[21] Yazdanpanah M, Assi C \& Shayan Y. 2011. Cross-layer optimization for wireless mesh networks with smart antennas. Computer Communications, 34(16): 1894-1911.

[22] YUAN J, Li Z, YU W \& Li B. 2006. A cross-layer optimization framework for multihop multicast in wireless mesh networks. IEEE Journal on Selected Areas in Communications, 24(11): 2092-2103, nov. 